\title{
Prevalence of opisthorchiasis in the Pavlodar region of the Republic of Kazakhstan
}

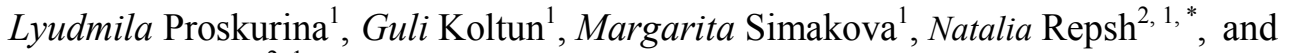 \\ Alexander Belov, ${ }^{2,1}$ \\ ${ }^{1}$ Primorskaya State Academy of Agriculture, pr. Blyukhera, 44, Ussuriysk, Primorsky Territory, \\ 692510, Russia \\ ${ }^{2}$ Far Eastern Federal University, ul. Sukhanova, 8, Vladivostok, Primorsky Territory, 690090, Russia
}

\begin{abstract}
The territory of the Pavlodar region is a natural focus with a high intensity of the spread of opisthorchiasis invasion among people. One of the important preventive measures in the foci of opisthorchiasis is the elimination of helminths in the body of the main owners, which leads not only to the rupture of the biological chains of helminth development, but also to the protection of the environment from invasive pollution. Opisthorchiasis is widespread in the Pavlodar region, the extensiveness of invasion among carnivores is $5.7 \%$. Cases of the incidence of cats were detected in five out of 10 districts (Aksu, Aktogay, Pavlodar, Shcherbaktinsky, Maysky), dogs - in two districts (Terenkol, Irtysh). Opisthorchiasis was not revealed in the study of the pig population. Cats and dogs, especially in coastal rural settlements of the region, support the existence of opisthorchiasis foci and contribute to their stabilization. The main source of the release of opisthorchis eggs into the external environment are cats, their infection rate is 2 times higher than that of dogs. In the Pavlodar region, opisthorchiasis of animals is caused by the $\mathrm{O}$. felineus pathogen or cat fluke.
\end{abstract}

\section{Introduction}

Fish is often the source of infestation in humans and domestic animals. Many of these fish species are target ones and valuable raw materials for the food industry. According to regulatory requirements, one of the most important characteristics of assessing the quality of fish products is parasitic safety (Technical Regulations ... 040/2016) [1]. Living in natural conditions, fish acquire parasites, some of which pose a danger to human health [2].

The relevance of studying opisthorchiasis is determined by the large spread of this invasion, significant economic damage, the development of severe complications in various diseases (cancer of the liver and pancreas, disorders of intestinal biocenosis, etc.), the development of immunodeficiency states, the combination of chronic opisthorchiasis with other infectious and non-infectious diseases, the development of various psychosomatic disorders and so on. [3, 4].

The territory of the Pavlodar region is a natural focus with a high intensity of the spread

* Corresponding author: repsh_78@mail.ru 
of opisthorchiasis invasion among people [5].

According to the data of V.I. Gershun and R.K. Tuyakov, who were engaged in the study of parasites in animals, the features of natural conditions and the peculiarity of the hydrological regime of the Pavlodar region were established, which ensure the persistent existence of foci of opisthorchiasis [6].

The lack of systemic and large-scale projects in the study of opisthorchiasis, both at the regional and national levels, does not fully resolve the issues of diagnosis, prevention and treatment of this pathology.

It has been studied that opisthorchia trematodes have the ability to involve the organs of the hepatobiliary system and the gastrointestinal tract in the pathological process: stomach, duodenum, pancreas and liver, and in $90 \%$ of cases, the main affected organ is the liver. But, at the same time, it has been established that this helminth does not cause damage to a single organ, but to the entire organism as a whole, since one of the mechanisms of the pathogenesis of the disease is the sensitization of the affected organism by enzymes and metabolites of larval forms and mature parasites, their repeated exposure, which in the aggregate can have an immunosuppressive effect [7].

The aim of the study: to study the prevalence of opisthorchiasis in the Pavlodar region of the Republic of Kazakhstan.

When analyzing the state of morbidity of the population with parasitic diseases, it was revealed that one of the socially significant problems of Kazakhstan and Pavlodar region is opisthorchiasis. More than 40 thousand people with opisthorchiasis are registered annually in all regions of Kazakhstan, and there is no trend towards a decrease in the incidence [8, 9].

One of the important preventive measures in the foci of opisthorchiasis is the elimination of helminths in the body of the main owners, which leads not only to the rupture of the biological chains of helminth development, but also to the protection of the environment from invasive pollution [10].

Opisthorchiasis is caused by two types of fluke helminths: Opisthorchis felineus and Opisthorchis viverrini.

The development of opisthorchis occurs with a threefold change of hosts: the first intermediate host is mollusks, the second intermediate is freshwater fish of the carp family (bream, carp, tench, dace, ide, roach, etc.), and the final one is mammals (cat, dog, fox, otter, arctic fox, sable, man) feeding on fish. The final hosts release eggs with larvae into the external environment along with feces. Gettinig into the water body, the eggs are swallowed by freshwater mollusks of the Codiella genus, in the body of which they undergo changes: first, miracidium emerges from the egg, which subsequently turns into sporocyst, redia and cercaria. The tailed larva (cercariae) emerges from the body of the mollusk and attaches to the body of cyprinids, penetrates into the connective and muscle tissue, where it encysts, turning into metacercaria. Being in the body of fish, after 6 weeks, the larvae become invasive, i.e. they acquire the ability to induce opisthorchiasis in their final hosts [11-13].

The analysis of the incidence of opisthorchiasis in 10 regions and 2 cities of the Republic of Kazakhstan (Almaty and Nursultan) shows an uneven distribution of the incidence of opisthorchiasis across the territories of individual regions and districts.

It has been established that the centers of opisthorchiasis are limited by the Irtysh River basin and its tributaries. High rates of human morbidity were registered in Pavlodar, medium - in West Kazakhstan and Kostanay regions, low - in North Kazakhstan, Akmola, Karaganda and East Kazakhstan regions. In some districts, large foci of opisthorchiasis adjoin each other, creating a single zone of the disease [14].

According to the data of the sanitary and epidemiological service of the Pavlodar region, opisthorchiasis is one of the most common parasitic diseases in the region. In 
addition to humans, domestic carnivores - cats, dogs, pigs - play an important role in the spread of opisthorchiasis.

Table 1 reflects information on the identification of people infected with opisthorchiasis in the Pavlodar region for 2017-2019.

Table 1. The number of opisthorchiasis diseases registered in the population of Pavlodar region for 2017-2019.

\begin{tabular}{|c|c|c|}
\hline Years & $\begin{array}{c}\text { Total number of } \\
\text { patients }\end{array}$ & $\begin{array}{c}\text { Including children } \\
\text { under 14 years old }\end{array}$ \\
\hline 2017 & 443 & 63 \\
\hline 2018 & 516 & 71 \\
\hline 2019 & 47 & 9 \\
\hline Total for 3 years & 1006 & 143 \\
\hline
\end{tabular}

In general, from 2017 to 2019,1006 patients with opisthorchiasis were registered in the Pavlodar region, including 143 cases of the disease in children under 14 years of age.

The largest number of patients occurs in 2018, which may be due to favorable climatic conditions for the reproduction of intermediate hosts, freshwater mollusks, which in turn caused massive infection of additional hosts - cyprinids, which, accordingly, gave the highest percentage of infection of final owners, firstly, people.

Data analysis revealed certain patterns of opisthorchiasis infection. The territorial division of the incidence of opisthorchiasis in children under 14 years old in the Pavlodar region, in general, coincides with the data for Kazakhstan, but draws attention to the fact that the incidence of children in the Pavlodar region is higher than in other places. On the one hand, this is due to the best medical supervision of children compared to adults, i.e. the best detection of conditions for opisthorchiasis, and on the other, non-compliance with preventive measures for children. In general, in Kazakhstan over the past year, there has been an increase in the incidence of children under 14 years old by $27 \%$, mainly due to the Pavlodar region.

In the total number of opisthorchiasis morbidity in the Pavlodar region, the population of the city - $723(71.8 \%)$ - exceeds rural residents - $283(28.1 \%)$. However, the main danger of opisthorchiasis infection is still professional: opisthorchiasis is mainly recorded among fishermen and public catering workers.

In addition, the lack of proper control over the spontaneous trade in fish that has not passed veterinary control and caught from the water bodies of the zone contaminated with fecal wastewater leads to a high percentage of morbidity in the fish-eating population.

The danger of infection with opisthorchiasis increases in the summer, when there is a large concentration of tourists near the water bodies [5, p. 17, 15, 16].

Medical, social and epidemiological factors affecting the formation and activity of foci of parasitic invasion are the nature of the diet, concomitant diseases - pathology of the digestive system, age, housing and living conditions [17].

\section{Materials and methods}

The work was carried out on the basis of the research laboratory "Parasitology" of the State Institution "Veterinary Directorate of Pavlodar Region".

The object of the study was the feces of animals (cats, dogs, pigs) with a verified diagnosis of chronic opisthorchiasis.

Feces were studied by the flotation method: using a saturated solution of sodium hyposulfite (1750 g per 1 liter of boiled water) - according to Shcherbovich.

A total of 229 animals were examined, including 130 cats, 56 dogs, and 43 pigs. 
In order to differentiate the invasive outbrake in domestic animals, studies were carried out using the method of complete helminthological autopsy according to K.I. Skryabin. In total, 10 bodies of cats and 10 bodies of dogs were examined.

\section{Research results}

Table 2 shows the results of studies of domestic animal feces for the presence of opisthorchus eggs.

From the results of the studies obtained, it is clear that invasive opisthorchiasis $(\mathrm{O}$. felineus) was detected in 13 animals out of 229 , which amounted to $5.7 \%$.

Table 2. Infestation of domestic carnivores with opisthorchiasis in the territory of Pavlodar region.

\begin{tabular}{|c|c|c|c|}
\hline Animal & \multirow{2}{*}{$\begin{array}{c}\text { Studied animal } \\
\text { type }\end{array}$} & \multicolumn{2}{|c|}{ Infected with O. felineus } \\
\cline { 3 - 4 } & feces, specimen & heads & EI, \% \\
\hline Cats & 130 & 11 & 8.5 \\
\hline Dogs & 56 & 2 & 3.6 \\
\hline Pigs & 43 & 0 & 0 \\
\hline Total: & 229 & 13 & 5.7 \\
\hline
\end{tabular}

For the study, cats were selected from the yards of local fishermen, where their pets regularly ate fish caught in local waters. When examining 130 cats, 11 cases of opisthorchiasis were found, which amounted to $8.5 \%$, with an invasion rate of 1 to 86 eggs.

The results of the analysis of the data obtained showed that cats infected with opisthorchiasis were found in five surveyed areas. The highest rates of infection were observed in Aksu (15.8\%) and Aktogay (10.3\%) districts. In the Pavlodar region, the incidence of cats was 3.4\%, in Shcherbaktinsky - 7.4\%, in Maysky - 7.7\%. The obtained data show that the difference in the level of distribution of opisthorchiasis in cats depends on the number of water bodies located there. Opisthorchiasis of dogs was detected only in Terenkol (6.3\%) and Irtysh (4.8\%) districts (Fig. 1).

In the study of the pig population, opisthorchiasis was not revealed, which indicates the absence of fish from local water bodies in their diet.

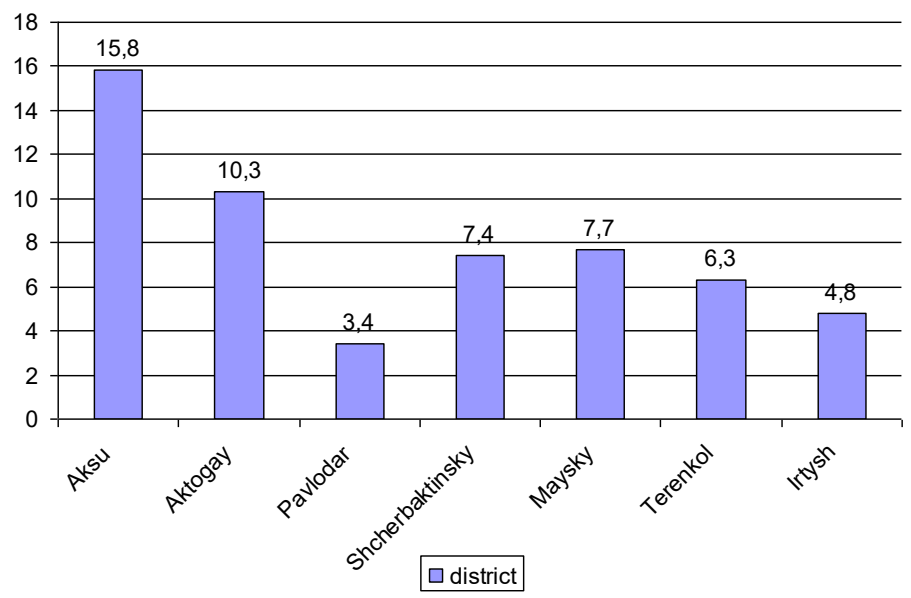

Fig. 1. The incidence of animals with opisthorchiasis in the districts of Pavlodar region. 
When studying the role of domestic carnivores in the distribution of invasive material in opisthorchiasis, it was found that cats are the main source of release of opisthorchis eggs into the external environment, their infection rate is 2 times higher than that of dogs.

Thus, we believe that cats and dogs, especially in coastal rural settlements, support the existence of opisthorchiasis foci and contribute to their stabilization.

When examining the liver and biliary tract, opisthorchiasis was registered in two cats $(2 \%)$ and one $\operatorname{dog}(1 \%)$. The study of the morphology of the parasite gave grounds to assert that in the Pavlodar region, opisthorchiasis of animals is caused by the $\mathrm{O}$. felineus pathogen or cat fluke.

\section{Conclusion}

From the results of the study, it was revealed that opisthorchiasis is widespread in the Pavlodar region, the extensiveness of invasion among carnivores is $5.7 \%$. Cases of the incidence of cats were detected in five out of 10 districts (Aksu, Aktogay, Pavlodar, Shcherbaktinsky, Maysky), dogs - in two (Terenkol, Irtysh). In the study of the pig population, opisthorchiasis was not revealed.

Consequently, cats and dogs, especially in coastal rural settlements of the region, support the existence of opisthorchiasis foci and contribute to their stabilization.

The main source of the release of opisthorchis eggs into the external environment are cats, their infection rate is 2 times higher than that of dogs.

In the Pavlodar region, opisthorchiasis of animals is caused by the $\mathrm{O}$. felineus pathogen or cat fluke.

\section{References}

1. O.Yu. Busarova, G.G. Koltun, V.V. Podvalova Parasites of salmon fish (Salmonidae) of Azabache Lake (Kamchatka), dangerous to human health and affecting the quality of fish raw materials Research of aquatic biological resources of Kamchatka and the north-western part of the Pacific Ocean, 48, 31-42. (2018)

2. O.Yu. Busarova, G.G. Koltun, V.V. Podvalova Assessment of the parasitic safety of salmonids from Lake Azabache, Kamchatka The role of agrarian science in the development of forestry and agriculture in the Far East: materials of the II National (All-Russian) scientific and practical conference, 56-58 (2018)

3. M.Sh. Akbaev, F.I. Vasilevich, P.M. Akbaev Parasitology and invasive diseases of animals: Textbook, 778 (2008)

4. H. Ross, A. Paiboon Sithithaworn, T. N. Petney Opisthorchis viverrini: an underestimated parasite in world health Trends in Parasitologis, 24, I. 11, 497-501 (2008)

5. A.I. Pantyukhov Opisthorchiasis in Pavlodar region: Epidemiology, clinic, struggle: thesis. 28 (1973)

6. V.I. Gershun, R.K. Tuyakova Workshop on veterinary hygiene, 263 (2017)

7. Thanh Thi Ha Dao, Tuan Van Bui et al. Opisthorchis viverrini infections and associated risk factors in a lowland area of Binh Dinh Province, Central Vietnam, 157, 151-157 (2016)

8. B.K. Zhumabekova Parasites of fish of the Kazakhstan Irtysh region: thesis, Ph.D. in Biological sciences, 40 (2009) 
9. A.S. Kusainova Epidemiological features of opisthorchiasis at the present stage: thesis, Ph.D. in Medicine., 25 (2010)

10. J. YilChai, K. Darwin Murrell, A. J. Lymbery Fish-borne parasitic zoonoses: Status and issues International Journal for Parasitology, 35, I. 11-12,1233-1254 (2005)

11. R.T. Safiullin, S.K. Shibitov Opisthorchiasis of carnivores, 12, 30-33 (2012)

12. D. J. Diemert 119 - Cestode and Trematode Infections, Infectious Diseases (Fourth Edition), 2, 1032-1037. (2017)

13. S.A. Beer Biology of the causative agent of opisthorchiasis, 336 (2005)

14. N.E. Beisenbieva Clinical and epidemiological characteristics of chronic opisthorchiasis and new approaches to etiotropic therapy(2016)- $89 \mathrm{p}$.

15. D.Yu. Ignatov Epizootological monitoring in parasitosis of dogs. Epizootological parameters of parasitosis of dogs (2007) 25p.

16. V.E. Polyakov, A.Ya. Lysenko Helminthiasis in children and adolescents (2003)

17. S. Tangkawattana, P. Tangkawattana Chapter Three - Reservoir Animals and Their Roles in Transmission of Opisthorchis viverrini Advances in Parasitology, 69-95. (2018) 